\title{
La consolidación de un modelo gramatical escolar en la enseñanza secundaria argentina (1863-1936)
}

\author{
Esteban Lidgett \\ Universidad de Buenos Aires, CONICET*
}

\begin{abstract}
Resumen
El propósito de este trabajo es analizar, desde una perspectiva que se inscribe en el marco teórico de la historiografía lingüística (Koerner 1974, 2007; Swiggers 1983, 2009), la configuración de la gramática escolar en la escuela secundaria argentina entre 1863 y 1936 . Se intenta proponer, en tal sentido, una hipótesis de periodización consistente con los datos aportados por las investigaciones previas sobre el corpus gramatical escolar en la Argentina (Calero Vaquera 2009, Lidgett 2011, Toscano y García y García Folgado 2012), a partir de un enfoque que privilegia aspectos de la historiografía externa (Swiggers 1983) hasta ahora no abordados por la bibliografía crítica. De esta forma, se avanza sobre la descripción del marco normativo (leyes, planes de estudio y programas) que condiciona el desarrollo de la gramática como disciplina escolar desde sus orígenes en 1863 hasta la primera intervención del Instituto de Filología en la programación escolar en 1936.
\end{abstract}

* Para correspondencia, dirigirse a: Esteban Lidgett (ealidgett@filo.uba.ar), Instituto de Lingüística, 25 de mayo 217/221 (CP 1002), tel. (54 11) 4342-9710/9718, int. 103. 
Palabras clave: historiografía lingüística, gramática escolar, periodización.

\author{
The Consolidation of a School Grammar Model in \\ Argentinean Secondary Education (1863-1936)
}

\begin{abstract}
The aim of this paper is to analyze, from a perspective that fits into the framework of linguistic historiography (Koerner 1974, 2007; Swiggers 1983, 2009), the configuration of the school grammar in Argentina between 1863 and 1936. In that sense, It intends to propose a periodizing hypothesis consistent with the data provided by previous researches on the school grammar corpus in Argentina (Calero Vaquera 2009, Lidgett 2011, Toscano and García and García Folgado 2012) from an approach that emphasizes a context-oriented historiography (Swiggers 1990), which has not been not addressed by the critical literature until now. Therefore, this paper advance in the description of the regulatory framework (laws, curricula and programs) that affects the development of school grammar discipline from its origins in 1863 to the first intervention of the Instituto de Filología in the school curricula in 1936.
\end{abstract}

Keywords: Linguistic historiography, school grammar, periodization.

Recibido: 31/08/2016 Aceptado: 13/03/2017

\title{
1. INTRODUCCIÓN
}

Las investigaciones que, en el marco global de la historia de la lingüística, han abordado de manera directa o indirecta los manuales de gramática escolar publicados entre fines del siglo XIX y principios del XX han privilegiado, en función de sus perspectivas, distintos aspectos de este objeto. Así, en primer lugar, existe un extenso conjunto de investigaciones que han considerado la gramática escolar en relación con el desarrollo de ciertas políticas lingüísticas tendientes a regular el uso del lenguaje (cf. Arnoux y Luis 2003; Blanco 1995, 2005; Di Tullio 2002; López García 2012; Vázquez Villanueva 2006). En estos trabajos se ha privilegiado en general el estudio de las políticas de Estado en torno a la cuestión lingüística $\mathrm{y}$, ocasionalmente, su articulación en el sistema educativo en general y en la 
enseñanza de la lengua materna en particular. En segundo lugar, desde una perspectiva cuyo desarrollo es bastante reciente en la Argentina, los trabajos vinculados a la reconstrucción historiográfica del corpus gramatical escolar de este país han abordado este objeto focalizando sobre todo la descripción interna de los manuales escolares para deslindar las tradiciones lingüísticas presentes en ellos (cf. Calero Vaquera 2008, 2009, 2010; Lacanna 2011; Lidgett 2011, 2012; Toscano y García y García Folgado 2012). En esta línea, se ha considerado en general la idea de que las gramáticas escolares son receptoras y transmisoras de las teorías lingüísticas y que, por lo tanto, su lugar en la escala epistemológica (es decir, el hecho de que sean instrumentos pedagógicos orientados a la enseñanza escolar y no académica) no debe constituir un motivo para su exclusión del análisis historiográfico.

De esta forma, desde diferentes perspectivas metodológicas y con objetivos distintos, ambos enfoques han puesto de relieve la pertinencia de la gramática escolar como objeto de investigación. No obstante los avances en ese sentido, la escasa sistematización del corpus gramaticográfico de la Argentina ${ }^{1}$, así como la carencia de una periodización que tuviera en cuenta la conformación de la disciplina escolar en relación con los objetivos pedagógicos y las exigencias sociales vinculadas con ellos, han sido impedimentos significativos a la hora de avanzar sobre el análisis historiográfico de la corriente gramatical escolar. Atendiendo a estas consideraciones, proponemos en este trabajo una aproximación a la configuración de la gramática escolar en la Argentina entre 1863 y 1936, con el objetivo de aportar una periodización maximalista (Swiggers 1983) que sirva de base para futuras investigaciones. En este sentido, presentamos un abordaje de los distintos instrumentos legislativos (planes de estudio, programas, leyes de educación, etc.) que intervienen en la configuración de la gramática desde los inicios del sistema nacional de enseñanza secundaria en 1863, hasta la reforma decisiva de los contenidos gramaticales que tiene lugar en 1936, con la intervención del entonces director del Instituto de Filología de la Universidad de Buenos Aires, Amado Alonso. Consideramos que este extenso periodo, que abarca más de setenta años en la historia de la enseñanza de la lengua en los colegios nacionales, se articula en tres etapas sucesivas que se distinguen en cuanto a la definición de objetivos

\footnotetext{
El proyecto de investigación grupal (PIP-CONICET) “La configuración de la gramática escolar argentina (1863-1922)", dirigido por Salvio Martín Menéndez y Guillermo Toscano y García y en el que esta investigación parcialmente se integra, se ha propuesto en su primera etapa el objetivo de establecer un corpus representativo de la producción gramatical del período.
} 
pedagógicos específicos, que procuramos identificar, y a la articulación de estos objetivos con los contenidos exigidos para la formación lingüística de los alumnos.

\section{MARCO TEÓRICO Y CONCEPTUAL}

Puesto que en este trabajo nos ocupamos centralmente de la configuración de la gramática como disciplina en la escuela secundaria, consideramos pertinente una especificación terminológica. Hablaremos de gramática escolar en un sentido acotado, refiriéndonos tanto a la tradición escolar de la educación media como a las obras escritas para ese nivel educativo. En este sentido, asumimos, siguiendo a García Folgado (2005), que independientemente de la función didáctica - que tiene un lugar significativo en la historia de la gramática (cf. Gómez Asencio 1985)- se habla de gramática escolar cuando desde un sistema educativo centralizado se piensa en un conjunto de contenidos que constituyen la enseñanza de la lengua nacional. Una definición de este tipo permite encontrar una especificidad analítica que trasciende el mero dato empírico de si una gramática constituye un libro escolar o no, es decir, si ha circulado o no en dicho ámbito. En otras palabras, para hablar de gramática escolar es necesario pensar en un sistema educativo que considera la gramática como disciplina que se propone consolidar una lengua nacional.

Desde el punto de vista de la historiografía interna, también se habla de gramática escolar para referirse a un cuerpo de doctrinas más o menos homogéneas que pueden rastrearse en un corpus de obras producidas por y para la enseñanza escolar (García Folgado 2013). No obstante, desde una perspectiva externa, la constitución de la gramática como disciplina escolar no constituye necesariamente un proceso tan uniforme y homogéneo. En ese sentido, cualquier aproximación historiográfica que asuma un estudio contextualizado tendrá necesariamente que considerar la gramática en tanto que disciplina escolar en el marco más general de formación lingüística que la institución escolar dispone ${ }^{2}$. Como intentaremos mostrar en este

2 Hablaremos de formación lingüística para referirnos al conjunto de contenidos que la programación escolar dispone para la enseñanza de la lengua materna, de las extranjeras y de las clásicas. Si bien estos contenidos exceden lo propiamente gramatical, pues abarcan otro tipo de tradiciones (por caso, la retórica), creemos que una consideración general de la formación 
trabajo, la constitución de una disciplina escolar constituye un proceso en el que intervienen diferentes actores y que, fundamentalmente, se encuentra articulado con la definición de los objetivos pedagógicos que los distintos proyectos educativos y el conjunto de la sociedad otorgan a la institución escolar. Estos objetivos determinan en términos generales cuáles son los saberes que deben privilegiarse en la formación de los estudiantes y, por lo tanto, intervienen de manera decisiva en la constitución de una disciplina escolar y en la definición de sus contenidos.

El objetivo central de este trabajo, como enunciamos más arriba, consiste en aportar una hipótesis de periodización. Al respecto, consideramos necesaria una aclaración metodológica respecto a los criterios de periodización con los que opera el historiador de la lingüística. Swiggers (1983: 69) define la periodización como "una división en periodos históricos, concebidos como unidades discretas de trabajo". Se trata de una operación metodológica a partir de la cual se construye un objeto ideal en la delimitación respecto de los otros objetos. De acuerdo con Swiggers, existen al menos dos tipos de periodización: "un tipo minimalista, que propone una estructuración extrínseca en el tiempo, y un tipo maximalista, que periodiza sobre una base intrínseca". La primera supone utilizar la periodización como hipótesis de trabajo puramente pragmática, mientras que la segunda implica, en cambio, justificar la periodización buscando características específicas para cada periodo, logrando una segmentación cronológica y tipológica a la vez. En ambos casos, la periodización funciona como un marco regulador para el historiador, como una hipótesis inicial de trabajo.

No obstante, la diferencia entre estos dos tipos de periodización es determinante: mientras que el primero parte de un criterio completamente externo y ubica el objeto de análisis en una línea temporal previamente concebida, el segundo va definiendo las etapas del devenir histórico como hipótesis de trabajo a medida que construye un objeto de estudio que no es independiente del contexto histórico. Creemos, en ese sentido, que solo una periodización del segundo tipo (maximalista) puede hacer justicia a la naturaleza integral de la perspectiva historiográfica que debe ser, siguiendo a Swiggers (1990) y Vila Rubio (2001), a la vez interna - esto es, orientada

lingüística en los colegios nacionales resulta indispensable para tener una noción del peso relativo que la tradición gramatical asume en el currículo escolar de cada etapa respecto del resto de los contenidos. 
al contenido- y externa -es decir, orientada al contexto ${ }^{3}-$. Tal perspectiva es la que procuramos seguir en este trabajo.

\section{UNA POSIBLE PERIODIZACIÓN}

Las investigaciones realizadas hasta ahora sobre el campo de la gramática como disciplina de enseñanza en la Argentina coinciden en señalar que las primeras obras gramaticales destinadas a la enseñanza del idioma en este país datan de 1817 (cf. Arnoux 2012). No obstante, si aceptamos que no puede hablarse de gramática escolar sino hasta que desde un sistema centralizado se piensa en un conjunto de contenidos que constituyen la enseñanza de la lengua nacional, entonces su punto de partida debe buscarse en el proceso de centralización que comienza a desarrollarse en la Argentina a partir de 1863, cuando en el contexto del periodo de la organización nacional se crea por decreto del presidente Bartolomé Mitre el Colegio Nacional de Buenos Aires (CNBA), sobre la base del antiguo Colegio de Ciencias Morales. El gobierno de Mitre inicia de esta forma la organización del sistema de la enseñanza secundaria a nivel nacional que continúa inmediatamente con la fundación de colegios nacionales en varias provincias del territorio nacional. De esta forma, la nacionalización de la educación, prevista ya en la primera Constitución Nacional de 1853, constituye un proceso que comienza a darse en el ámbito de la instrucción secundaria y que continuará progresivamente hasta alcanzar también a la escuela primaria.

La periodización que proponemos en este trabajo tiene su origen, por lo tanto, en este decreto inicial que constituye el comienzo de la organización a escala nacional del sistema de educación media. Desde el punto de vista de la organización del sistema educativo, existen numerosas obras que han propuesto distintas posibles periodizaciones, cuyas inflexiones se encuentran determinadas por la emergencia de diferentes planes de estudio, por la organización del currículo escolar, por la presencia de proyectos de

\footnotetext{
$3 \quad$ Al respecto, señala Swiggers (1990: 22): "It is clear, then, that there are important links between content and context of theories, links which are thus not only relevant to the fact that particular theories appear at a certain time, but also to the message carried by the theory. Notwithstanding these links, a twofold approach of the history of linguistics -as a succession of formal thought-contents and techniques coupled with them, and as the development of a 'linguistic culture'- seems justified".
} 
reformas integrales o, sencillamente, por la asunción de nuevos ministros en el área (Dussell 1997, Puiggrós et al. 1991, Solari 1949, Tedesco 1972). No obstante, desde nuestra perspectiva resulta productivo articular los distintos proyectos educativos con cambios sustanciales en el modo de comprender la enseñanza del idioma y, en particular, la configuración de la gramática. Por este motivo, hemos propuesto un tipo de periodización que considera tres etapas, cuya conformación obedece a la preponderancia de un modelo determinado de enseñanza con consecuencias palpables en el campo de la gramática escolar.

De esta forma, consideramos como primera etapa la que comienza en 1863, a partir del decreto del presidente Mitre que da origen a un proyecto nacional de educación de orientación enciclopedista y cuyos efectos más visibles en el desarrollo de la gramática escolar pueden verse en la incorporación del análisis lógico a los contenidos generales de la enseñanza del idioma. El primer punto de inflexión lo constituye la reforma de Amancio Alcorta en 1884, cuyos lineamientos de base positivista alentarán la definición de un proyecto de educación secundaria de orientación utilitarista que tendrá consecuencias notables en la configuración de la gramática escolar argentina. La tercera y última etapa comienza con la reforma de Juan Carballido en 1891 y marca la consolidación definitiva de una tradición gramatical-cuyas características analizaremos oportunamente-presente en la programación curricular y en los manuales escolares al menos hasta la década de 1930. El punto culminante de esta periodización lo constituye la reforma de 1936, articulada sobre la base del proyecto de reforma integral elaborado por Juan Mantovani en 1934. Se trata de la primera reforma en la que las autoridades del Instituto de Filología toman parte activa en la reelaboración de los contenidos de la gramática escolar, lo que constituye un punto de inflexión decisivo para la enseñanza de la gramática en los colegios nacionales.

En las secciones siguientes, por lo tanto y como anticipamos en la introducción, desarrollamos una caracterización de cada una de las etapas que hemos consignado en este acápite.

\subsection{Primera etaPa: el MOdelo enCiClopedista (1863-1884)}

El decreto de 1863 que establece la creación del CNBA sobre la base del Colegio Seminario de Ciencias Morales será el primer paso hacia la nacionalización de la educación secundaria. Esta tendencia centralizada se evidencia en los decretos sucesivos con fecha del 9 de diciembre de 1864, a partir de los cuales se crean los colegios nacionales de San Juan, Tucumán, 
Mendoza, Catamarca y Salta. Cada uno de ellos explicita en su artículo segundo que "la enseñanza durará cinco años y se ajustará al Programa de estudios que rige en el Colegio Nacional de Buenos Aires" (apud García Merou 1901: 130).

El plan de estudios propuesto en el decreto fundacional del CNBA, cuya elaboración había sido encargada a Amadeo Jacques, estipulaba cinco años de educación secundaria y dividía los estudios en tres áreas: Letras y Humanidades, Ciencias Morales y Ciencias Exactas. En lo que respecta a la enseñanza de la lengua materna incluía la asignatura "Idioma castellano", con unidades dedicadas a la "gramática explicada, ortología, prosodia y ortografía". Asimismo, la formación lingüística se ampliaba con estudios en literatura española, lengua latina y tres lenguas extranjeras (francés, inglés y alemán). La presencia de la lengua latina como contenido indispensable para la formación humanística es notable; su estudio se complementaba con contenidos de "Gramática comparada" y ejercicios de traducción oral y escrita de "los principales autores latinos" (cf. García Merou 1901).

La distribución de los contenidos por año de estudio exhibe con claridad la preeminencia de una formación humanística $\mathrm{y}$, en particular, con una marcada orientación a los estudios clásicos. En los cinco años de estudio, el único contenido que se mantiene constante es el estudio del latín. En lo concerniente al castellano, proponía un programa gradual que partía de la enseñanza de la gramática, con especial énfasis en el aprendizaje de la ortografía, para avanzar luego hacia el desarrollo de las habilidades de la lectura, la composición y la elocución. Se trata del modelo clásico para la formación de oradores, modelo que se articula con una de las finalidades características del proyecto educativo de esta etapa: la formación de una elite destinada a dirigir la nación.

En síntesis, los datos relevados por la planificación educativa contenida en la legislación y por los programas del CNBA -que constituían el modelo al que debía ajustarse el resto de los colegios nacionales- muestran la consolidación de un esquema de enseñanza gramatical que, a pesar de ciertas alteraciones menores, permanece constante entre 1863 y 1884. En ese sentido, si como sostiene Chervel (1991) las disciplinas escolares se construyen en la intersección entre los objetivos educativos y los contenidos teóricos que determinan la instrucción, puede decirse que la etapa que va de 1863 a 1884 manifiesta un modelo de enseñanza de la lengua que sigue ciertos parámetros regulares. Desde el punto de vista de los objetivos de enseñanza, el objetivo central de la formación lingüística pautada por el proyecto fundacional de Mitre consistía en formar una clase dirigente apta para ejercer las funciones de gobierno (cf. Blanco 2005). En la programación de los contenidos, ese objetivo se articuló en un currículo que priorizaba 
la instrucción en lenguas clásicas y extranjeras; y, en particular, para el estudio de la lengua materna, destacaba la formación literaria y promovía la ejercitación de la escritura. Las reformas de la década de 1870, llevadas adelante siguiendo los lineamientos del proyecto inicial ideado por Sarmiento y Avellaneda, no implicaron modificaciones sustanciales respecto de esta primera configuración de la gramática escolar.

El modelo de enseñanza gramatical que se configura durante esta etapa inicial manifiesta en general un debate entre los antiguos modelos memorísticos, asociados a la enseñanza en la educación primaria, y los nuevos esquemas didácticos propuestos sobre todo por el análisis lógico y la teoría gramatical de Bello (cf. Blanco 2005). Esa oscilación, exacerbada por la carencia de material didáctico de la que adolecían los nuevos colegios, comienza a inclinarse del lado de los renovadores hacia mediados de la década de 1870, cuando se amplía la circulación de compendios sobre la gramática de Bello (cf. Blanco 1995). En efecto, el programa oficial de 1874, redactado por Isaac Larraín, exhibe el estudio de la gramática en sus cuatro partes tradicionales (analogía, sintaxis, prosodia y ortografía) y deja ver, en la parte dedicada a la analogía, la influencia de Bello, al proponer la "clasificación de las palabras según sus distintos oficios en el discurso" (CNBA 1874). La tradición filosófica francesa también se hace presente en el texto que incluye, dentro de la unidad dedicada a la sintaxis, el análisis lógico y el estudio de las proposiciones. Finalmente, puede observarse en el programa toda una unidad dedicada a la composición, lo que constituye una característica de esta primera etapa de la gramática escolar en la que la tradición retórica exhibe un mayor peso relativo respecto de la gramatical.

\subsection{Segunda etapa: el MOdelo Positivista (1884-1891)}

El plan de estudios propuesto por Amancio Alcorta en 1884 se inscribe en un proceso general de reformas educativas que alcanza todos los niveles de la educación nacional. En ese sentido, la reforma que el Ministerio le encarga a Alcorta, rector del CNBA entre 1883 y 1890, apunta a establecer un nuevo esquema para la educación nacional, caracterizado sobre todo por la penetración del positivismo (cf. Solari 1949, Tedesco 1972). En lo que respecta a la formación lingüística, el nuevo modelo positivista se caracterizó particularmente por disminuir o eliminar la formación clásica y acentuar la enseñanza de modelos gramaticales que apuntaran a la depuración de los vicios.

La primera renovación que promueve este plan consiste en plantear una división entre cursos regulares, obligatorios para todos los establecimientos, 
y cursos libres, que serían de carácter facultativo. Los cursos regulares mantenían la duración de seis años que había sido establecida en la reforma de 1870. En lo que respecta a la formación lingüística, el plan de estudios implantaba algunos cambios considerables. En sintonía con el carácter nacional que Alcorta pretende otorgarle al nuevo plan de estudios, la asignatura que en los planes de la década anterior se denominaba "Idioma castellano", pasa a nombrarse ahora "Idioma nacional"4.

Si se compara con el plan de estudios anterior, el de 1879, puede observarse, además del cambio de nombre de la asignatura, un incremento de dos lecciones semanales adicionales para el total del año, lo que constituye un total de 13 lecciones semanales. Asimismo, dentro de los contenidos mínimos que se detallan en el plan de estudios, en los dos primeros años se reemplazan los ejercicios de lectura razonada y el análisis lógico gramatical por la enseñanza de la gramática en sus cuatro partes tradicionales: analogía, sintaxis, prosodia y ortografía.

Si se analizan los planes de estudio y programas de "Idioma nacional" entre 1884 y 1888, puede observarse que la disposición de los contenidos establecida presenta un estudio de la lengua que durante los dos primeros años de enseñanza se reduce al aprendizaje de la gramática dividida en sus cuatro partes tradicionales: analogía, sintaxis, ortología y ortografía. Este ordenamiento parece seguir la disposición tradicional presente también en las obras de referencia para la enseñanza de la gramática en la Argentina: la Gramática de Bello y la de la Real Academia Española. Esta nueva organización tuvo como consecuencia el desplazamiento de los contenidos vinculados con la composición, que había sido el eje fundamental de la enseñanza del castellano en la etapa anterior.

El programa analítico del CNBA fue elaborado por los profesores José Hidalgo Martínez y Emilio Castro. Hidalgo Martínez será, además, autor del Curso gradual de gramática castellana (ca. 1884), un importante manual escolar que contará con 19 ediciones hasta el año 1930 (cf. Calero Vaquera 2009). El programa, que sigue el eje de esa gramática, hace una propuesta heterogénea que busca combinar la tradición bellista con la académica. Así, por ejemplo, en la segunda unidad sobre analogía se encuentran como

\footnotetext{
4 Respecto de esta denominación, Arturo Costa Álvarez señala que "aplicada a la lengua general de nuestro país fue en su origen una expresión pedagógica oficial". No obstante -agrega- "las denominaciones tradicionales de la asignatura vuelven de tiempo en tiempo a nuestros planes de estudios en el orden nacional: tenemos «idioma nacional» en los decretos de 1884, 1887, 1888, 1898 y 1900; «lengua castellana» en el de 1886; «idioma castellano» en los de 1891 y 1893; e «idioma patrio» en los de 1901 y 1902" (1922: 100-101).
} 
contenidos la clasificación semántica de las palabras, propia de la tradición académica, y la clasificación "por el oficio que desempeñan en la oración", que se acerca a la propuesta de Bello. También en la unidad XIV sobre tiempos verbales se explicita la "Comparación de la conjugación llamada americana y la de la Academia Española" (CNBA 1884).

La presencia de Andrés Bello y la Academia española como fuentes inexorables de las gramáticas escolares en el mundo hispánico decimonónico ha sido señalada con frecuencia (Álvarez Martínez 1994, Calero 2009); sin embargo, lo que resulta característico de este programa y de la gramática de Hidalgo Martínez es el hecho de que aportarán por primera vez una matriz estructural sobre la que se construirán los numerosos tratados gramaticales y programas de las décadas siguientes. En efecto, si la reforma de Alcorta establece, al menos en el ámbito del estudio del idioma, una dirección general para la planificación educativa sucesiva, el modelo gramatical que exhibe Hidalgo Martínez aportará el modelo macroestructural para la elaboración de gramáticas pedagógicas del español en la Argentina para las cuatro décadas siguientes. Se trata de un esquema básico en el que se establece que el saber lingüístico necesario para los colegios secundarios consiste en la adquisición de una base sólida de cada una de las cuatro partes tradicionales de la gramática, distribuidas en los dos primeros años de estudio. A ese esquema se le agregará ocasionalmente un tercer año de literatura preceptiva o, a partir de 1891, de lingüística y etimología. De esta forma, no obstante la presencia de cierta heterogeneidad en las fuentes documentales de los distintos manuales escolares, puede decirse que, desde el punto de vista de la planificación educativa, será esta configuración de la gramática la que se imponga a partir de la reforma de 1884, desplazando el modelo de formación ilustrada propio de las décadas anteriores.

La consolidación de la gramática (considerada en sus cuatro partes tradicionales) como eje vertebral de la organización curricular de los cursos de "Idioma nacional" constituye una característica central de la etapa que estamos analizando. En efecto, la inserción del paradigma positivista en los colegios nacionales a partir de la década de 1880 supuso la introducción de una concepción utilitarista de la educación secundaria que, en el ámbito específico de la enseñanza de la lengua materna, se articuló en torno a la intensificación de una formación gramatical estrictamente normativa que fue paulatinamente desplazando el modelo analítico de tradición francesa que se había cultivado en la etapa anterior (cf. Blanco 2005). En ese sentido, en un contexto social atravesado por la cuestión inmigratoria, la formación lingüística, tanto en la recientemente centralizada escuela primaria como en los colegios nacionales, se reorganizó en esta etapa con el objetivo de dotar al ciudadano de una cultura del idioma destinada a estigmatizar los 
usos vulgares que se distanciaban de la norma culta (cf. Di Tullio 2004, López García 2009).

Esta nueva configuración de la gramática escolar se hace explícita en la programación curricular. El programa de "Idioma nacional" del CNBA en 1887 estipula para el primer año el estudio de la ortografía y ortología y destaca la necesidad de corregir la pronunciación de los estudiantes. Así, por ejemplo, entre los contenidos del programa se detallan:

XXVII: De la correcta pronunciación de las consonantes. Vicios que se comenten en la pronunciación de las letras B y V, C, S y Z, D, H, J, Ll e Y, N, S, I e Hi, X. Preceptos que deben aplicarse para corregirlos. Eufonía. Cacofonía. Vicios antieufónicos [...].

XXXIIII [...] Palabras que de ordinario se pronuncian mal por cambio, supresión o agregación de vocales o consonantes (CNBA 1887).

Estas exigencias se trasladaron rápidamente a los textos gramaticales que, a partir de 1880, se multiplicaron en el territorio nacional. Hidalgo Martínez, por ejemplo, en su obra gramatical (1930: 285), acuña el término "barbarismo prosódico" para designar la acción de "faltar a la correcta pronunciación", ya sea por "articulación viciosa de las letras", por la utilización de "vulgarismos" (es decir "la mala pronunciación de las sílabas") o la incorrecta acentuación. Este tipo de prácticas escolares prescriptivas de los usos lingüísticos, como sostiene López García (2012: 125), acentuó la distancia entre las prácticas orales de la sociedad y el modelo normativo a través del cual el sistema educativo pretendía homogeneizar la lengua nacional; pues, con contadas excepciones, las gramáticas escolares del periodo en su afán hípercorrectivo censuraron varios usos propios de la variedad rioplatense como el seseo, el yeísmo o el voseo (cf. Delgado 1947, López García 2009).

\subsection{TERCERA ETAPA: LA CONSOLIDACIÓN DEL MODELO GRAMATICAL ESCOLAR}

La siguiente reforma al plan de estudios se produce en 1891, en el contexto de la fuerte agitación política y social que siguió a la denominada Revolución del Parque de 1890 que provocó la renuncia del presidente Miguel Juárez Celman. En el ámbito educativo, el levantamiento liderado por la recientemente formada Unión Cívica tuvo consecuencias notables que, de acuerdo con Tedesco (1972), en los años sucesivos provocarían un drástico cambio en la orientación del proyecto educativo ideado por la oligarquía gobernante. En ese contexto, el nuevo gobierno de Carlos Pellegrini encarga al Ministerio de Instrucción Pública una nueva reforma al plan de estudios que permita resolver los que considera problemas todavía persistentes: la 
articulación de la enseñanza primaria, secundaria y superior, y la reducción de contenidos innecesarios en las asignaturas. En nuestra periodización, esta reforma constituye un punto de inflexión a partir del cual comenzará a consolidarse un modelo de formación gramatical que permanecerá vigente, con breves alteraciones, hasta 1936.

El nuevo plan de estudios es precedido por una circular que el ministro Juan Carballido publica en el diario La Nación el 21 de abril de 1891 y que tiene como destinarios a las distintas autoridades de los colegios nacionales y a la sociedad en su conjunto. En esta circular, Carballido (1891: III) explica los fundamentos sobre los que debe articularse el nuevo proyecto educativo y destaca, sobre todo, la necesidad de resolver la situación de desintegración provocada por la afluencia inmigratoria:

Tan violenta ha sido la avenida inmigratoria, que podría llegar a absorber nuestros elementos étnicos. Están sufriendo una alteración profunda todos nuestros elementos nacionales: lengua, instituciones prácticas, gustos e ideas tradicionales. A impulso de este progreso spenceriano -que es realmente el triunfo de la heterogeneidad-debemos temer que las preocupaciones materiales desalojen gradualmente del alma argentina las puras aspiraciones, sin cuyo imperio toda prosperidad nacional se edifica sobre arena.

De acuerdo con Carballido, la corrupción social que provoca la "avenida inmigratoria" abarca también y, especialmente, el ámbito de la lengua. En ese sentido, el autor advierte que la "lengua común" constituye el mayor vínculo con las demás naciones hispanoamericanas y que en ningún otro país como en la Argentina esa lengua común se ve tan seriamente amenazada por el peligro de la dialectización. Si bien la percepción del problema que significaba la inmigración para el devenir de la lengua cuenta con testimonios anteriores en el ámbito educativo nacional, la circular de Carballido manifiesta por primera vez con claridad la posición de las autoridades ministeriales en lo que respecta a la cuestión del idioma y a la necesidad de instrumentar políticas educativas tendientes a conservar la "pureza de la lengua".

Es ese el contexto en el que se presenta el informe de la Comisión encargada de sugerir la reforma de los planes de estudio en 1891. Dicho informe también se hace eco de las preocupaciones del ministro acerca de la lengua nacional:

La enseñanza del Idioma Castellano, que es el idioma nacional, es deficiente, comprobándolo así sus resultados. No obstante los cambios operados en los métodos y en los textos, ha podido notarse que, hechos todos los estudios, los alumnos carecían de un conocimiento completo 
del propio idioma, siendo sumamente defectuosas las aplicaciones que debían hacerse en los cursos superiores (apud Fernández 1903: 920).

La expresión de estas preocupaciones en torno a la enseñanza del idioma, presente tanto en la circular de Carballido como en el informe de inspección de 1891, constituye un dato novedoso en la legislación educativa que venimos relevando. En efecto, estos documentos ponen de manifiesto el lugar destacado que las autoridades ministeriales le otorgan a la formación lingüística, a la que ubican en el centro de la programación educativa, poniendo de manifiesto su función político-lingüística: la depuración de la lengua.

La centralidad que asume la enseñanza de la lengua en la instrucción general se evidencia también en la ampliación de la formación clásica propuesta por el nuevo plan, lo que parece retomar la vertiente enciclopédica del plan de Mitre. Al respecto, el ministro Carballido sostiene:

Filológicamente, somos herederos directos de los latinos, mucho más que los franceses y los mismos italianos [...]. De este parentesco directo se deducen dos consecuencias pedagógicas; desde luego, es evidente que, para entender a los autores -no decimos ser latinistas- único objetivo actual de la enseñanza, debemos con un buen método requerir mucho menos tiempo que los demás pueblos, sin excluir los otros neolatinos [.... La otra consecuencia pedagógica a que aludí es un corolario de la primera. Siendo el menor esfuerzo necesario, efecto de parentesco estrecho entre ambas lenguas, es evidente que su esfuerzo simultáneo es de alta conveniencia y provecho para el dominio de una y otra" (1891: XXI-XXII; el destacado es del autor).

La segunda de las consecuencias pedagógicas a las que alude Carballido es central para comprender el lugar que a partir de entonces pasará a ocupar el latín en los planes de estudio. Su enseñanza se adelanta y comienza en el segundo año, para que pueda ir a la par de la de la lengua materna, con cuyos contenidos se complementa. Esta correlación entre el latín y la enseñanza de la lengua materna comporta también la inclusión por primera vez de los contenidos de lingüística histórica que serán presentados como un área en la que confluyen ambos saberes.

El plan de 1891 vuelve al esquema de cinco años, transformando el sexto en un primer año preparatorio que se dictará en cada facultad. Como veremos, este plan resulta trascendental para la configuración de la gramática escolar argentina, pues los programas que se ajustan a su organización introducen por primera vez contenidos de "Lingüística" que se ajustan a la tradición teórica de la gramática histórico-comparada. Como hemos procurado demostrar en 
un trabajo anterior (Lidgett 2012), esta tradición, junto con la académica y la filosófica, constituyen las tres fuentes fundamentales sobre las que se organizarán los contenidos mínimos de enseñanza gramatical a partir de 1891 y, al menos, hasta 1936. En efecto, en lo que respecta a las cuatro partes tradicionales de la gramática (analogía, sintaxis, prosodia y ortografía) la influencia de la Real Academia Española irá progresivamente desplazando a Bello, que en los nuevos manuales solo es mencionado marginalmente. Por su parte, la tradición filosófica, presente -como vimos-en la gramática escolar argentina de los primeros años, añade a los programas el análisis lógico como contenido mínimo que permanecerá a lo largo de las diferentes reformas. Finalmente, a partir de los planes de estudio de 1891, la gramática histórico-comparada tendrá su lugar en los programas y manuales escolares, consolidándose como un conjunto definido de contenidos mínimos que aparecerán bajo la nómina "Lingüística".

En síntesis, la reforma de 1891 muestra en general una tendencia progresiva hacia la recomposición del modelo humanista, sustentado en los primeros planes de estudios de los colegios nacionales, frente al modelo utilitarista de la enseñanza científica positivista. La consecuencia más notable para el modelo gramatical escolar consiste en la reincorporación del análisis lógico, la intensificación de los estudios literarios como base para el estudio de la gramática, el restablecimiento de las prácticas de escritura y oralidad (composición y elocución) y, en lo que respecta a la formación lingüística en general, la restitución de los contenidos sobre lengua latina y literatura clásica. En particular, a partir de la reforma de 1891, la planificación educativa exhibe la necesidad de articular la formación clásica con la enseñanza de la lengua materna. En ese sentido, resulta interesante notar que los contenidos sobre lingüística histórica ("nociones de etimología", "estudio histórico de la lengua"), que aparecen por primera vez en el plan de 1891, se incluyen con el argumento de articular la enseñanza clásica con la gramatical. La presencia de este contenido será defendida sobre todo por profesores de latín, entre los que destacan las figura de Matías Calandrelli, autor del primer diccionario filológico comparado publicado en la Argentina; y Baldmar Dobranich, autor de la primera gramática histórica del español publicada en este país ${ }^{5}$. Su inclusión en los programas de "Idioma castellano" y en

\footnotetext{
5 Baldmar Dobranich (1853-1912), nacido en Gibraltar, se traslada a la Argentina en 1886 para desempeñarse como docente de inglés en el Colegio Nacional de Buenos Aires. En la Argentina, junto con Matías Calandrelli (1845-1919), será un impulsor de los estudios de lingüística histórica y comparada (véase Ricci 1922). En este sentido, resulta significativa su Gramática histórica de la lengua castellana ([1893] $\left.{ }^{3} 1900\right)$, que constituye el primer
} 
las compilaciones de gramática permanecerá estable, según la formulación impuesta por el propio Dobranich, desde 1892 hasta 1936.

\section{El plan Garro: hacia un periodo de estabilidad}

La estabilidad de los planes de estudio y la necesidad de una reforma integral siguieron siendo ejes de debate en las polémicas educativas durante los primeros años del siglo XX. Hacia 1910, el ministro Rómulo Naón encarga al entonces rector del CNBA, Enrique de Vedia, una investigación sobre el estado de la enseñanza secundaria. Para ello, de Vedia convoca a un gran número de docentes de distintos colegios del territorio nacional con el objetivo de que emitan sus opiniones en torno a una serie de cuestiones que considera fundamentales para la enseñanza media. A la hora de publicar las conclusiones de su investigación, que incluyen una síntesis de las opiniones vertidas por los docentes, de Vedia (1910: 14) reconoce los resultados de la escuela secundaria mitrista, pero sostiene:

[...] sobrevinieron luego los reformistas, los europeístas inconscientes y desordenados, por lo mismo; surgió un normalismo enciclopédico, que ultrapasando los límites de su función primaria, invadió el campo de la escuela media para errar a cada golpe; se implantaron formas exóticas e incongruentes; creóse la funesta correlación de estudios, engendradora del enciclopedismo educacional del que nació la vacua superficialidad del presente, - un presente que encierra veinte años largo-, a una reforma siguió otra, agravando el mal que se quería curar con deslumbrantes descubrimientos pedagógicos o sociológicos [...].

El problema generado por esta sucesión de reformas experimentales que juzga incongruentes sería resuelto, según de Vedia (1910: 15-16), "el día que tengamos la Ley Orgánica correspondiente, que dé estabilidad al plan,

antecedente de una obra escolar de lingüística histórica publicada en la Argentina. Dicha gramática servirá, como sostiene Delgado (1947), como fuente indispensable para la sección de "Lingüística" presente en los programas de castellano y en los textos oficiales desde 1892, cuando el propio Dobranich incluye estos contenidos en el programa del Colegio Nacional de Buenos Aires que él mismo redacta según lo dispuesto por la reforma del año anterior (cf. Dobranich 1892). Esta obra, que en lo esencial sigue los postulados de García Ayuso (1871) y Monlau (1856) (cf. Lidgett 2012), constituye un documento esencial para analizar la vinculación entre los estudios clásicos y la formación gramatical que es promovida por las reformas de 1888 y 1891 y que, como veremos, dejará su huella en la gramática escolar al menos hasta la década de 1930 (cf. Lidgett 2015). 
estabilidad a los programas [...], en oposición a la variabilidad de todo eso que ha sido la causa única del desastre educacional que palpamos".

Los resultados de esta investigación son acogidos por la siguiente administración, la del ministro Juan Garro, que se hace cargo de la cartera de Educación en octubre de 1910. Durante su gestión, Garro promueve una serie de reformas sucesivas que intentan renovar la enseñanza media. En primer lugar, decreta la creación de una nueva Dirección General de Enseñanza Secundaria, que viene a reemplazar a la antigua Inspección General de Enseñanza, y que se encargará en lo sucesivo de promover las reformas de los planes de estudio, entre otras tareas administrativas y de inspección. Posteriormente, estipula nuevos requisitos para la designación del personal docente, que hasta entonces no necesitaba de un diploma para ejercer la enseñanza ${ }^{6}$.

Finalmente, el 12 de febrero de 1912, Garro decreta la nueva "Organización de la Enseñanza Secundaria General". El nuevo plan establece una enseñanza que "debe ser integral y bastarse para sus fines" y debe encaminarse a "suministrar a la mayoría de los habitantes de la Nación los conocimientos necesarios para actuar eficazmente en la vida individual o colectiva, con prescindencia de toda orientación hacia profesiones o carreras determinadas" (MJIP 1913: 12). Esta declaración contradice toda la concepción utilitaria de la educación que se había impuesto durante la segunda etapa (1884-1891).

En ese sentido, el nuevo plan de estudios recupera también la enseñanza del latín con el argumento de que su enseñanza es "accesible a los jóvenes" y, asimismo, que "es incontestable su utilidad" porque "aparte de ser el lenguaje de la ciencia [...] se halla ligado con el castellano, que más que un idioma distinto, según se ha hecho notar, es el mismo latín transformado en sus elementos fonológicos y morfológicos por las variaciones que introdujeran en él los diversos pueblos que le hablaron" (1913: 16). De esta forma, Garro retoma los argumentos de Carballido y promueve la necesidad de articular la enseñanza del castellano con la del latín.

La similitud con el plan de Carballido puede verificarse también en otros aspectos que se vinculan con la enseñanza de la lengua materna. En efecto, el plan vuelve a incluir los contenidos de gramática histórica ("nociones 
de lingüística y etimología”), necesarios para articular con el latín, y hace referencia también a la "depuración y enriquecimiento del vocabulario y de las formas", lo que supone nuevamente una tendencia a la función correctiva y depuradora de la gramática. Los contenidos desglosados del nuevo programa de castellano permiten observar una confluencia de distintas tradiciones previas. Si el retorno de los contenidos de lingüística y etimología y la mención de la "depuración" del vocabulario acercan este programa al de 1892, la insistencia en la inducción de las reglas y la presencia de la composición lo aproximan al de 1863. En ese sentido, es posible sostener que este programa, que se mantendrá vigente con leves modificaciones durante los siguientes 25 años, logra la estabilidad necesaria al amalgamar elementos de aquellos dos programas tan disímiles.

En las "instrucciones" que se detallan al final del programa, se insiste particularmente en que la "lectura razonada y la composición" son esenciales para la formación del alumno. Se establece que "cada quince días los alumnos deberán redactar en clase una composición sobre un tema propuesto por el profesor". Asimismo, se especifica que "la parte gramatical se ha de derivar, siempre que sea posible, de los ejercicios de lectura y de composición" (Ministerio de Justicia e Instrucción Pública 1913: 47), lo que constituye un triunfo de los métodos pedagógicos propuestos por los normalistas. Otro aspecto destacable de este programa, y que verifica nuevamente su carácter heteróclito, es la presencia de Bello entre los contenidos de analogía, a quien se menciona en particular con relación a su clasificación de tiempos verbales. En la bibliografía sugerida, se menciona a la Academia, Bello/ Cuervo, Bello, Benot, Cejador, Monlau y Menéndez Pidal, cuya inclusión resulta toda una novedad.

\section{La reforma de Mantovani}

Si bien el impulso reformista presente entre las distintas autoridades ministeriales vuelve a hacerse presente en los años posteriores a la administración de Garro, durante el periodo radical (1916-1930) se logra una estabilidad relativa en la organización de la educación secundaria, que permanece por esos años bajo el influjo del proyecto humanista encarnado en el plan de educación general y preparatoria de Garro. En el ámbito de la enseñanza de la lengua materna, la estabilidad del plan Garro supone, aunque con algunas salvedades, la permanencia de la tradición gramatical que ya se encontraba cristalizada en los colegios secundarios argentinos: una gramática escolar que se establece en el programa de 1892 y se reformula en el de 1912. Puede decirse que, a pesar de las diferencias oportunamente señaladas entre ambos programas, los dos constituyen articulaciones similares de una 
enseñanza del idioma concebida para los fines del proyecto humanista de formación general y enciclopédica.

El proceso que conduce a la reforma de 1936, que pone fin a esta tercera etapa, se inicia en octubre de 1933, cuando se conforma una comisión encargada de elaborar nuevos planes de estudio para toda la enseñanza secundaria. La comisión encargada de elevar los planes de los colegios nacionales y liceos de señoritas estaba integrada por Ernesto Nelson, Lydia Peradotto, José Rezzano, Ricardo Levene, Juan Nielsen, René Bastianini y Francisco Romero. En los considerandos de la propuesta, la comisión se manifiesta a favor de una orientación general de la enseñanza hacia lo que denominan "humanidades modernas", en oposición a las "humanidades clásicas" ya perimidas. Las "humanidades modernas" se organizarían en torno a la enseñanza de las lenguas vivas: la lengua materna y algún idioma extranjero. En ese sentido, el proyecto señala el carácter formativo de los idiomas extranjeros por su utilidad como "instrumento de adquisición de saber", lo que permitiría articularlos también con la enseñanza de otras disciplinas y, en particular, con el castellano, para cuyo aprendizaje sería útil puesto que estimula "la claridad y precisión en la manifestación de las ideas" (Mantovani 1934: 134).

En 1934, el entonces Inspector General de Enseñanza Secundaria, Juan Mantovani, retoma estas consideraciones de la comisión y busca concretar una reforma integral. El nuevo plan se hacía eco de toda la tradición humanista anterior y pretendía intensificar todavía más la formación en letras. Para la organización de la educación secundaria consideraba dos ciclos: uno inferior de cuatro años y otro superior de dos. En ese plan, el castellano (que, según se detalla, abarcaba "lengua, gramática y literatura") se extendía a lo largo de los dos ciclos, sumando un total de 34 horas semanales; el idioma extranjero (a elección entre francés o inglés) ocupaba un total de 18 horas semanales para los seis años. La formación clásica, por su parte, permanecía ausente.

La reforma, sin embargo, no llega a concretarse en 1934. No obstante este primer fracaso, la reforma de los programas de castellano se produce finalmente en 1936, cuando se reúne una comisión especial, integrada entre otros por Amado Alonso y Pedro Henríquez Ureña, con el fin de renovar los contenidos de esta asignatura ${ }^{7}$. Esta reforma será crucial por

\footnotetext{
La dimensión rupturista de esta reforma en la que participa el Instituto de Lingüística a través de las figuras de Amado Alonso y Pedro Henríquez Ureña ha sido señalada en numerosas investigaciones (Battista y Lidgett 2015; Bombini 1995-96, 2004; Manacorda de Rosetti 1995-96).
} 
dos motivos: en primer lugar, porque la participación de Amado Alonso, director del Instituto de Filología de la Universidad de Buenos Aires desde 1927, expresa la primera intervención eficiente de un organismo profesional y especializado como el Instituto de Filología en la elaboración de los contenidos escolares; en segundo lugar, y en consonancia con el anterior, porque el nuevo plan implica la traslación de ese modelo profesional de la filología a la enseñanza media, y esto no solo porque supone un modificación radical de los contenidos, sino también en la medida en que logra alterar las condiciones establecidas para la formación de profesores. Hasta ese momento, en la Argentina no se requería de un título habilitante específico para enseñar el idioma castellano. Si bien, como hemos visto, a partir de las reformas de Garro se había votado un reglamento que estipulaba que para dictar clases en los colegios nacionales se requería diploma, el mismo reglamento aceptaba que esta condición podía suplirse si se contaba con cinco años de antigüedad en la docencia secundaria o universitaria o si se contaba con méritos equivalentes. Así, en la práctica, la enseñanza del castellano todavía continuaba siendo ejercida por profesionales de cualquier disciplina. Si bien este aspecto de la reforma no interesa directamente al tema de este trabajo, su mención permite esclarecer la dimensión de los cambios implicados.

Al mismo tiempo, la labor de Alonso al frente del Instituto de Filología había supuesto un incremento de las actividades de transferencia desde esta institución hacia la formación docente. En efecto, en 1932, un año antes de que se conformara la asamblea de profesores, Alonso imparte una serie de conferencias en el Colegio Libre de Estudios Superiores de Buenos Aires, con el título "Clases de palabras y categorías gramaticales". El curso, dictado para profesores de castellano, expone una crítica a los diversos sistemas de clasificación de palabras y sostiene la necesidad de retomar el criterio sintáctico-funcional de Bello para su implementación en la enseñanza de la lengua (cf. Toscano García et al. 2013). Será en esas clases, en la redacción de los programas reformados y en la Gramática castellana que escribe junto con Henríquez Ureña (1938-39) donde será posible observar la intervención de Alonso en la reformulación de la tradición gramatical en la Argentina.

Si observamos el programa vigente en 1937, el año posterior a la reforma, y lo comparamos con los expuestos anteriormente, podremos ver la dimensión de los cambios efectuados. Los nuevos programas de la asignatura de Castellano abarcan, como los anteriores, los tres primeros años de la enseñanza media. Cada año está distribuido en 10 unidades que combinan prácticas de gramática, lectura y composición; asimismo, cada año contiene apartados especiales donde se explica cómo enseñar determinadas subdisciplinas, qué evaluar en cada caso y cuál es la bibliografía indicada 
para el profesor. Estas indicaciones dan cuenta de un programa analítico más completo desde el aspecto pedagógico, probablemente como consecuencia de las reformas introducidas también en las asignaturas pedagógicas de los profesorados (Bombini 2004). Sin embargo, el aspecto más saliente de esta reforma no está en la organización macroestructural de la asignatura, que muchas veces antes había cambiado sin alterar en nada la tradición gramatical, sino en los contenidos mismos. En ese sentido, la presencia del criterio sintáctico-funcional de clasificación de palabras que Alonso reclamaba en sus conferencias de 1932 es el eje articulador de la enseñanza gramatical en el programa.

La tradición de la enseñanza gramatical suponía exclusivamente para el primer año temas de analogía, ocasionalmente acompañados de nociones de ortología, ortografía, lectura y composición. A partir de la reforma de 1936, la clásica división de la gramática en sus cuatro partes ya no volverá a ser el eje sobre el cual se articulen los contenidos. En el programa de 1937, en efecto, se señalan los siguientes temas para las tres primeras unidades:

La oración. Articulación primera de la oración en sujeto y predicado. Predicado verbal y nominal. El sustantivo como núcleo del sujeto y el verbo como núcleo de la predicación. En qué consiste la concordancia del sujeto con el verbo.

Señalar, sobre textos y sobre expresiones orales, las articulaciones posibles del sujeto: sustantivo con adjetivo; con preposición y otro sustantivo $[\ldots]$.

Señalar las articulaciones posibles del predicado verbal: verbo con adverbio; con sustantivo como complemento directo, como complemento indirecto y como complemento circunstancial. El predicado nominal: sustantivo o adjetivo con verbo copulativo o sin él (CNBA 1937).

Como se ve, el punto de partida y eje articulador de la gramática es ahora la sintaxis. El conocimiento de la sintaxis es la base para establecer las funciones de las distintas clases de palabras y por ello pasa a estar por primera vez como contenido dentro del primer año de estudios. Durante el segundo y tercer año se intensifica su estudio a partir de la enseñanza de la coordinación y la subordinación. Existen otros contenidos gramaticales que se alternan en las distintas unidades: el estudio de la fonética, incluida por primera vez en los programas, la ortología, la ortografía, la lectura y la composición. Asimismo, es preciso mencionar que también desaparecen definitivamente del programa los contenidos vinculados con la tradición filosófica francesa (clasificación lógica de las proposiciones, teoría del verbo único, etc.). Finalmente, existe un cambio notable en la bibliografía que se recomienda a los profesores: el nuevo programa incluye una lista 
que contiene la Gramática de la lengua castellana de Bello, La oración y sus partes de Rodolfo Lenz, el Manual de pronunciación española y el Compendio de ortología de Tomás Navarro Tomás, el Manual de gramática histórica española de Ramón Menéndez Pidal y la Filosofía del lenguaje de Karl Vossler.

En ese sentido, la renovación de los programas de 1936, llevada a cabo con la participación de Amado Alonso y Pedro Henríquez Ureña, constituye un proceso de modernización de la tradición gramatical escolar en la Argentina que trastocará el sistema educativo de enseñanza media y terciaria y consolidará por primera vez una tradición profesional en la gramática escolar.

\section{CONCLUSIONES}

Hemos propuesto en este trabajo una periodización de la historia de la gramática escolar en la Argentina atendiendo a la articulación entre los diversos proyectos educativos y las configuraciones propuestas para la enseñanza de la lengua materna. El criterio con el que organizamos la exposición pretendió, de esta forma, encontrar los puntos de contacto entre las finalidades sociales con arreglo a las cuales se conforma la planificación educativa y la distribución de los contenidos vinculados con la formación lingüística y, en particular, con la enseñanza de la gramática.

En ese sentido, postulamos una primera etapa que abarca las distintas planificaciones presentes desde la organización de los colegios nacionales en 1863 hasta la primera reforma de orientación positivista en 1884 . Durante esa primera etapa, la educación secundaria se organizó siguiendo la orientación humanista y enciclopedista que originariamente le había impuesto Jacques en 1863. Si bien, como hemos analizado, durante la década de 1870 se sucedieron reformas tendientes a incorporar una educación menos elitista y más orientada a la formación práctica, la enseñanza gramatical se organizó bajo similares parámetros. En efecto, se tendió a una formación que incentivara las prácticas de lectura y escritura, incluyendo la composición como contenido estable durante toda la etapa; y se diseñó una enseñanza gramatical que pretendió renovar la doctrina tradicional, con la que se enseñaba en la escuela primaria, incorporando la obra de Bello, cuya implementación en los colegios nacionales impulsó la publicación de diversos compendios. Por su parte, la tradición filosófica, presente en la formación ilustrada de la juventud ya en las primeras obras gramaticales 
argentinas, incorporó hacia la década de 1870 el análisis lógico como contenido de enseñanza.

Esta configuración de la gramática escolar que responde al proyecto humanista que organiza la educación secundaria sufrirá una primera modificación cuando en 1884 Wilde proponga un cambio de rumbo para la educación nacional. La reforma de Wilde inicia, entonces, la segunda etapa en la configuración de la gramática escolar a nivel de los colegios nacionales. Por su orientación positivista, esta reforma propone limitar la enseñanza de las humanidades y, como consecuencia de ello, en la enseñanza del castellano queda suprimida la composición, que había sido un contenido característico de la formación lingüística previa. En un contexto social atravesado por la cuestión inmigratoria, el enfoque práctico de la educación se aplica a la enseñanza del idioma privilegiando la función prescriptiva de la gramática, antes que las prácticas de escritura. El modelo aportado por la Gramática de la Real Academia Española se impone sobre la tradición bellista, que de todas maneras permanece tangencialmente en los programas, y se instrumentan gramáticas escritas en Argentina que comienza ser editadas siguiendo el orden macroestructural aportado por los nuevos programas oficiales. El modelo de enseñanza de la gramática establece la analogía y la sintaxis, durante el primer año; la ortografía y la prosodia durante el segundo y la gramática general junto con la literatura preceptiva en el tercero. Esta permanencia de la gramática general da cuenta del grado de consolidación que la tradición filosófica exhibe en la historia de la gramática escolar argentina.

Finalmente, en el marco de una restauración de la tendencia humanista del proyecto educador, la reforma de 1891 encarada por Carballido vuelve a introducir una reconfiguración de la enseñanza del idioma. En el marco de la creciente preocupación por lo que una parte de las autoridades educativas consideraba el peligro de la desintegración lingüística producto del aluvión inmigratorio, Carballido promueve un nuevo plan en el que la enseñanza del castellano adquiere un lugar preponderante. Por primera vez, las autoridades ministeriales dejan constancia de la necesidad de proveer una enseñanza gramatical que se destine a la depuración de la lengua, un objetivo que, aunque de manera menos explícita, ya estaba presente desde la reforma de Wilde. La nueva programación para la enseñanza del castellano retoma entonces la función prescriptiva de la gramática, vuelve a incluir la composición (propia del currículo humanista), mantiene el análisis lógico y la gramática general y agrega, por último, los contenidos de lingüística y etimología que se incorporan con el argumento de articular la formación clásica con la gramatical. Las reformas sucesivas entre 1893 y 1915 no lograron desmontar esta configuración de la gramática escolar que, 
finalmente, encuentra el mayor periodo de estabilidad luego de la reforma de Garro que, con excepción de los métodos novedosos que introduce, no cambia en lo sustancial la estructura de los contenidos curriculares de 1891.

El análisis de los distintos programas que hemos reconstruido en este capítulo conduce a pensar que, a pesar de las distintas orientaciones exhibidas por las sucesivas reformas, en 1891 se llega a una configuración particular de la gramática escolar que permanece inalterable en lo sustancial hasta la reforma de 1936. Esa configuración, como hemos mencionado, parece sustentarse en una tradición propiamente escolar y autónoma, es decir, una tradición creada y organizada para servir como instrumento de enseñanza en los colegios secundarios. La síntesis sobre la que se consolida la gramática escolar en esta etapa comprende la asimilación de las distintas teorías lingüísticas que venían circulando en el ámbito escolar (como la teoría de Bello, la de la Real Academia Española o la de la tradición filosófica francesa) y la incorporación de otras (como la lingüística histórica) que son reelaboradas como contenidos curriculares en los nuevos programas.

No obstante, creemos que estas consideraciones preliminares que solo describen la gramática escolar desde su configuración curricular oficial deben ser examinadas en el marco más general de un análisis que comprenda la descripción comparativa de un corpus representativo de gramáticas escolares. La elaboración de este corpus y su análisis comparado son todavía una cuenta pendiente para la historiografía lingüística en la Argentina.

\section{REFERENCIAS BIBLIOGRÁFICAS}

Alonso, Amado y Pedro Henríquez Ureña. 1938-1939. Gramática castellana. Segundo curso. Buenos Aires: Librería "El Ateneo".

Álvarez Martínez, María Ángeles. 1994. La gramática española en América. La Laguna: Secretariado de Publicaciones de la Universidad.

Arnoux, Elvira y Carlos R. Luis (comps.). 2003. El pensamiento ilustrado y el lenguaje. Buenos Aires: Eudeba.

Arnoux, Elvira. 2012. La primera gramática escolar 'general' publicada en Buenos Aires en los años de la Independencia: la Gramática Española o Principios de la Gramática General aplicados a la Lengua Castellana de Felipe Senillosa. Histoire, Epistémologie, Langage XXXIV, 2: 43-61.

Battista, Emiliano y Esteban Lidgett. 2015. Clases de palabras y categorías gramaticales (1932): renovación de la tradición gramatical escolar en unas conferencias inéditas de Amado Alonso. Recherches 14. Culture et Histoire dans l'Espace Roman. Strasbourg: Université de Strasbourg.

Blanco, María Imelda. 1995. Reformulaciones de la Gramática castellana de Andrés Bello destinadas a la escuela media. Letterature d'America XV, 59: 87-127. 
2005. La enseñanza de la lengua nacional en los colegios secundarios (18631898). Tesis de maestría inédita. Maestría en ciencias del lenguaje. Instituto Superior del Profesorado "Dr. Joaquín V. González".

Bombini, Gustavo. 1995-1996. Reforma curricular y polémica: Amado Alonso y los programas de nivel secundario en la Argentina. Cauce: Revista de filología y su didáctica $\mathrm{N}^{\circ}$ 18-19. Centro Virtual Cervantes.

2004. Los arrabales de la literatura. La historia de la enseñanza literaria en la escuela secundaria argentina (1860-1960). Buenos Aires: Eudeba.

Calero Vaquera, María Luisa. 2008. Una muestra de la presencia en Hispanoamérica del análisis lógico y gramatical: el Tratado (Buenos Aires, 1880) de V. García Aguilera. Ed.Uco 3: 27-39.

2009. Apuntes sobre el Curso gradual de gramática castellana (ca. 1930) de José Hidalgo Martínez y su lugar en la tradición escolar argentina. Revista argentina de historiografia lingüistica I, 2: 151-174.

2010. Sintaxis y gramática escolar en la España del siglo XIX: su proyección en Hispanoamérica. En Ideias Linguísticas na Península Ibérica (séc. XIV a séc. XIX), editado por Carlos Assunção, Gonçalo Fernandes y Marlene Loureiro, vol 1, pp. 67-84. Münster: Nodus Publikationen.

Carballido, Juan. 1891. Plan de estudios y programas. Circular. Ministerio de Instrucción Pública (A los señores rectores de colegios nacionales). La Nación, Buenos Aires, 21 de abril.

Chervel, André. 1991. Historia de las disciplinas escolares. Reflexiones sobre un campo de investigación. Revista de Educación n 295: 59-111.

Colegio Nacional de Buenos Aires (CNBA). 1874. Programas del Colegio Nacional de Buenos Aires para los exámenes correspondientes al año escolar de 1874. Buenos Aires: Imprenta Americana.

1884. Programa de Gramática General y Razonada. Buenos Aires: Librería Rivadavia.

1887. Programa de Idioma Nacional. Buenos Aires: Librería Rivadavia.

1937. Programa de Idioma Nacional. Buenos Aires: Librería Rivadavia.

Costa Álvarez, Arturo. 1922. Nuestra lengua. Buenos Aires: Sociedad Editorial Argentina.

DE Vedia, ENRIQUe. 1910. Investigación sobre el estado de la enseñanza secundaria: informe oficial: Ministerio Naón. Buenos Aires: Talleres gráficos de la Penitenciaría Nacional.

Delgado, María Jesús. 1947. La enseñanza del castellano en los Colegios Nacionales de la Argentina. San Miguel de Tucumán: Facultad de Filosofía y Letras de la Universidad Nacional de Tucumán.

Di Tullio, Ángela. 2002. La otra crisis de la gramática escolar. En G. Parodi (ed.). Lingüistica e Interdisciplinariedad: Desafios del nuevo milenio. Ensayos en Honor a Marianne Peronard, pp. 281-295. Valparaíso: Ediciones Universitarias de la Universidad Católica de Valparaíso (Chile).

2004. Políticas lingüísticas e inmigración. El caso argentino. Buenos Aires: Eudeba.

Dobranich, Baldmar. 1892. Programa de Castellano para los Colegios Nacionales y las Escuelas Normales. Buenos Aires: Imprenta y litografía de T. Nettenoven e hijo.

[1893 ${ }^{3} 1900$. Gramática histórica de la lengua castellana. Nociones de Lingüistica y Etimología. Acompañada de ejercicios. Buenos Aires: Mariano Moreno.

Dussel, InÉs. 1997. Currículum, humanismo y democracia en la enseñanza media (1863-1920). Buenos Aires: FLACSO y Oficina de publicaciones del CBC, Universidad de Buenos Aires.

FERnÁNDEZ, JuAn RAMÓn. 1903. Antecedentes sobre la enseñanza secundaria y normal en la República Argentina. Buenos Aires: Ministerio de Justicia e Instrucción Pública. 
García Ayuso, Francisco. 1871. El estudio de la filología en su relación con el sanskrit. Madrid: Impr. y Estereot. M. Rivadeneyra.

García Folgado, Ma. José. 2005. La gramática española y su enseñanza en la segunda mitad del siglo XVIII y principios del siglo XIX (1768-1813). Tesis de doctorado. Universidad de Valencia.

2013. Los inicios de la gramática escolar en España (1768-1813). Una aproximación historiográfica. München: Peniope.

García Merou, Juan. 1901. Leyes, decretos y resoluciones sobre instrucción superior. Buenos Aires. Taller tipográfico de la Penitenciaría Nacional.

Garro, Juan Mamerto. 1913. La enseñanza secundaria. Decretos orgánicos, resoluciones de la dirección general, plan de estudios y programas analíticos. Buenos Aires: Talleres gráficos de la Penitenciaría Nacional.

Gómez Asencio, José Jesús. 1985. Subclases de palabras en la tradición española (1771-1847). Salamanca: Universidad de Salamanca.

Hidalgo Martínez, José. 1884. Compendio de la gramática de la lengua castellana. Buenos Aires: Librería Rivadavia de G. Mendesky.

1930. Curso gradual de gramática castellana adaptado al programa general de la asignatura de los Colegios Nacionales, Escuelas Normales y de Comercio. Buenos Aires: Ivaldi \& Checchi, editores.

Koerner, E. F. K. 1974. Editorial: Purpose and Scope of Historiographia Linguistica. Historiographia Linguistica 1, 1: 1-10.

2007. La historiografía de la lingüística. Pasado, presente y futuro. En Josefa Dorta, et. al. (eds.). Historiografía de la lingüística en el ámbito hispánico. Fundamentos epistemológicos y metodológicos, pp. 15-56. Madrid: Arco Libros.

Lacanna, Georgina. 2011. Tradición gramatical y concepciones lingüísticas en Idioma patrio. Una gramática elemental (1910), de Z. Vélez de Aragón. Revista argentina de historiografía lingüística III, 2: 87-108.

LidGETT, EsteBAn. 2011. Gramática escolar y políticas lingüísticas en la Argentina: un análisis de la Gramática de la lengua castellana (1893) de Baldmar Dobranich y Ricardo Monner Sans. En Revista argentina de historiografia lingüistica III, 2: 109-132.

2012. Los inicios de la gramática histórica en la Argentina: un estudio sobre la Gramática histórica de la lengua castellana (1900) de Baldmar Dobranich. En Historiografia lingüistica: líneas actuales de investigación, editado por Elena Battaner Moro, Vicente Calvo Fernández y Palma Peña Jiménez, vol. 2, pp. 549-558. Münster: Nodus Publikationen. 2012.

2015. Tradiciones gramaticales y discurso sobre la lengua nacional en la obra de Ricardo Monner Sans (1893-1926). Tesis doctoral inédita.

López García, María. 2009. Representaciones de la variedad rioplatense en los manuales de la escuela primaria en Buenos Aires. Tesis doctoral inédita.

2012. Implicancias glotopolíticas del estilo dialógico en los manuales escolares. Páginas de Guarda. Revista de Lenguaje, Edición y Cultura Escrita 11: 13-31.

MANacorda DE RosetTI, MABel. 1965. La gramática estructural en la escuela primaria. Buenos Aires: Kapelusz.

1968. La gramática estructural en la escuela secundaria: su valor formativo. Buenos Aires: Kapelusz.

1995-1996. Amado Alonso y el programa de castellano aplicado en la Argentina en 1936: una revolución copernicana, Cauce. Revista de filología y su didáctica 18-19: 417-433.

Mantovani, Juan. 1934. Proyecto de reformas a los planes de estudio de la enseñanza media. Buenos Aires: Taller tipográfico de la Penitenciaría Nacional. 
Ministerios de Justicia e Instrucción Pública (MJIP). 1913. Plan de estudios y programas para los Colegios Nacionales. Boletín oficial de la República Argentina XXI: 5713.

Monlau, Pedro Felipe. 1856. Diccionario etimológico de la lengua castellana. Madrid: Imprenta y estereotipia de M. Rivadeneyra.

Puiggrós, Adriana et al. 1991. Historia de la educación argentina. Buenos Aires: Galerna.

Ricci, Clemente. 1922. Discurso en homenaje a Baldmar Dobranich. Ethel Bordoli, Stella Maris Fernández et al. 1996. La investigación las bibliotecas y el libro en cien años de vida de la Facultad de Filosofia y Letras de la Universidad de Buenos Aires, pp. 27-35 Buenos Aires: Universidad de Buenos Aires.

Solari, Manuel Horacio. 1949. Historia de la Educación Argentina. Buenos Aires: Paidós.

Swiggers, Pierre. 1983. La méthodologie de l'historiographie de la linguistique. Folia Linguistica Historica IV/1: 55-81.

1990. Reflections on (Models for) Linguistic Historiography. En Werner Hüllen (ed.). Understanding the Historiography of Linguistics: Problems and Projects, pp. 2134. Münster: Nodus.

2009. La historiografía de la lingüística: apuntes y reflexiones. Revista argentina de historiografía lingüística I, 1: 67-76.

2012. Historiografía de la gramaticografía didáctica: apuntes metodológicos con referencia a la (historia de la) gramática española y francesa. En Neus Vila Rubio (ed.). Lengua, literatura y educación en la España del siglo XX, pp. 15-37. Berna: Peter Lang.

Tedesco, Juan Carlos. 1972. Educación y sociedad en la Argentina (1880-1945). Buenos Aires: Ediciones Solar.

Toscano y García, Gulllermo y María José García Folgado. 2012. Los textos de gramática en la escuela argentina (1863-1900). Escribas. Revista de Letras VII: 27-54.

Toscano y García, Guillermo, Emiliano Battista y Esteban Lidgett. 2013. "Les archives d'Amado (1896-1952): correspondance et travaux inédits", en Valentina Chepiga y Estanislao Sofía (eds.). Archives et manuscrits de linguistes: études et état des lieux. Lieja: Academia-Bruylant.

Vázquez Villanueva, Graciana. 2006. Una política lingüística en el callejón: Hacer la nación, unificar la lengua en Argentina (1890-1900). Revista Lenguaje 34: 97-123.

Vila Rubio, Neus. 2001. Historiografía lingüística: observaciones terminológicas y conceptuales. En Mariana Maquieira et al. (eds.). Actas del II Congreso Internacional de la Sociedad Española de Historiografía lingüística, pp. 1003-1013. Madrid: Arco Libros. 